Author version: IEEE Geosci. Remote Sens. Lett., vol.11(1); 2014; 54-58

\title{
Multifractal Approach for Seafloor Characterization
}

Bishwajit Chakraborty, Member, IEEE, K. Haris, G. Latha, Member, IEEE, Nicolas Maslov and Andrew Menezes

Abstract - This study involves multifractal characterization of pockmark seepage associated seafloor along the central part of the western continental margin of India (WCMI). Six representative blocks of backscatter and bathymetry co-registered image data were used to characterize the seafloor. Two distinct multifractal formalisms were applied to determine the characteristics. The first formalism employs data analyses using generalized dimension $D(q)$ and multifractal singularity spectrum $f(\alpha)$ linked shape parameters, based on the 'strange' attractors that exhibit multifractal scaling. The second approach is designed as 'stochastic' multifractal fields that connect the image block quantification to the three fundamental parameters namely, degree of multifractality $\alpha$, sparseness $C_{1}$ and degree of smootheness $H$. The present investigation using the two multifractal formalisms to characterize the seafloor backscatter and bathymetry data provides comparative results that can be expounded upon.

Index Terms - Multifractal, Multi-beam backscatter and bathymetry images, 'Strange' attractors, 'Stochastic' multifractal fields

B. Chakraborty, K. Haris, and A. Menezes are with the CSIR-National Institute of Oceanography, Goa: 403004, India (e-mail: bishwajit@nio.org)

G. Latha is with the National Institute of Ocean Technology (MoES), Chennai: 600100, India

N. Maslov is with the IRENav, Artset Metiers Paris Tech, Paris, France 


\section{INTRODUCTION}

The seafloor bathymetry and the associated backscattering data of submerged objects have an extremely wide range of spatio-temporal scales necessitating application of 'power law' to carry out the analyses. The 'power law' behavior in such instances requires multifractal analyses [1] in order to determine if both (seafloor backscatter and bathymetry image data) follow 'fractal' statistics. It is imperative to treat such data as a scale invariant field requiring multifractal measures and exponent functions, rather than a unique scaling exponent (such as fractal dimension) [2]. An infinite number of fractal dimensions would be needed to completely characterize the scaling, as evident in other fields like: satellite radiance data [3], medicine [4], and ecology [5].

The two important formalisms of multifractal analyses such as: i) 'strange' chaotic attractors [6] and ii) 'stochastic' multifractal fields [7] have been used in this study. The first formalism is based on the 'box counting' method. It involves analyses of multifractal distribution pattern using the correlation dimension $D(q)$, and multifractal spectrum $f(\alpha)$ related shape parameters [i.e., width of the spectrum $W$, degree of asymmetry $B$, and stability of spectrum $\Delta f(\alpha)$ ]. The properties of these functions at different statistical moments are used to characterize the spatial distribution of seafloor backscatter and bathymetry seepage blocks used in this study. The other formalism allows quantification of the seepage blocks with three fundamental parameters namely, degree of multifractality $\alpha$, sparseness $C_{1}$, and degree of smootheness $H$ [8].

Dandapath et al. [9] had reported seafloor seepages in this area (WCMI) using multi-beam echosounding (MBES) backscatter and bathymetry data. The investigations were related to underlying geology, pockmark occurrences, overlying sediment texture, and sediment movements due to strong influences of monsoonal bottom currents. Thereafter utilizing the method proposed by Seuront and Spilmont [10], Dandapath et al. [11] had noted the possible multifractal behavior of the MBES backscatter and bathymetry image data. Therefore, the present study involves quantitative estimation of the multifractal parameters from seafloor seepage to enhance the understanding of the processes in the area (WCMI).

The application of the two multifractal techniques could substantiate the hitherto applied numerical inversion based characterization [12],[13], and the soft computational technique based classification [14],[15], of the seafloor sediments employing the backscatter data. The section on 'material and methods' covers the study area, data set descriptions, and the implementation aspects of 
the multifractal techniques. The related section 'study results and discussion' provides the interrelationships among the estimated parameters in terms of seafloor morphology and is followed by 'conclusions'.

\section{MATERIALS AND METHODS}

\section{A. Study Area and Data Sets}

The backscatter and bathymetry data used in the present study were acquired from the central part of WCMI, where the water depth varies between 145-330m (Fig.1). Simrad EM 1002 MBES operating at $95 \mathrm{kHz}$ was used to acquire the data. The important morphological aspects of the study area have been investigated in detail, and 112 pockmarks related to the seepages were identified from the seafloor maps generated using ArcGIS [9]. The backscatter and bathymetry image blocks having $400 \mathrm{x}$ 400 pixels, were classified according to the degree of seepage based on the backscatter strength as well as fractal dimension (determined using box-dimension technique) [11]. The analyses carried over each gray tone image blocks (with digital numbers ranging 0 to 255) suggest that the area with very high seepage has higher fractal dimension and lower dimension with very low seepage. In this study, six representative seepage blocks i.e., F20, J19, F07, Q19, S23 and N25 having very high, high, moderate, low, very low, and no evidence of seafloor seepages respectively (Table 1) have been considered for analyses.

\section{B. 'Strange' Attractors - Multifractal Spectrum and Related Parameter Estimation}

Self-similar fractals are scale invariant i.e., possessing a structure with a basic characteristic of non-scaling. They can be divided into two categories. The first one is the monofractal, having strict geometric self-similarity that can be described with a single fractal dimension. The other is the multifractal that requires a series of fractal spectrum rather than a unique fractal dimension. The multifractal formalism based on the 'strange' chaotic attractors followed here identifies a set of parameters derived from the shape of such a fractal spectrum. As a part of the image analyses, the variation of these shape parameters among the seafloor seepage blocks is examined to measure the 'complexity' of the field. In view of this, a probability distribution is estimated using the box counting method [16]. The partition function $\chi(q, \varepsilon)$ that describes the probability of 'containing the object' (i.e., the values of backscatter and bathymetry in this application), within each box $i$, can be calculated for different moments of $q$ using: 


$$
\chi(q, \varepsilon)=\sum_{i=1}^{n(\varepsilon)} m_{i}^{q}
$$

where $m$ is the mass of the measure, $\varepsilon$ is the length of the box and $n(\varepsilon)$ is the number of boxes. Based on this, the mass exponent function $\tau(q)$ shows how the moments of the measure scales with the box size and is given as:

$$
\tau(q)=\lim _{\varepsilon \rightarrow 0} \frac{\log \chi(q, \varepsilon)}{\log (\varepsilon)}=\lim _{\varepsilon \rightarrow 0} \frac{\log <\sum_{i=1}^{n(\varepsilon)} m_{i}^{q}>}{\log (\varepsilon)}
$$

The generalized fractal dimension function $D(q)$ can then be calculated from $\tau(q)$ as $D(q)=\tau(q) /(q-1)($ where $q \neq 1)$. The singularity index $(\alpha)$ is subsequently determined to calculate the singularity spectrum $f(\alpha)$ by Legendre transformation of the $\tau(q)$ curve as $\alpha(q)=d \tau(q) / d q$. Finally the $f(\alpha)$, which represents the fractal dimension of the subset with the same singularity strength $(\alpha)$ is determined to describe the characteristic of the different hierarchy of fractal as $f(\alpha)=q \alpha(q)-\tau(q)$.

An image can be realized as multifractal when the graph of $\alpha$ vs. $f(\alpha)$ (i.e., multifractal spectrum), exists and has the shape of an inverted parabola. If the curve $f(\alpha)$ converges to a single point, it can be termed as monofractal wherein $D(q)$ is constant for all values of $q$. The width of the generalized dimension i.e., $\Delta D(q)=D\left(q_{\max }\right)-D\left(q_{\min }\right)$, is a measure of multifractality and indicates the deviation from monofractal behavior [16]. The particulars of the shape parameters used to describe the multifractality (based on the said formalism) are shown in Fig. 2.

In order to distinguish the multifractal spectrum $f(\alpha)$ quantitatively, it is convenient to calculate the width of the spectrum $W$ so as to measure the overall variability [Fig. 2(b)]. A wider $f(\alpha)$ spectrum is indicative of larger $W$, denoting multifractality. Such a situation reveals a 'heterogeneous' seafloor. In the case of a monofractal set, $W$ would be small and tending to 'zero'. The spectrum will converge to a single point signifying a 'homogeneous' seafloor. The other parameter $B$ measures the asymmetry of the curve and shows the dominance of low or high fractal exponents [17]. The value of $B$ is zero for symmetric shapes and positive or negative for right or left-skewed shapes respectively. A left-skewed spectrum denotes low fractal exponents dominating the distribution, while a right-skewed spectrum implies dominance of high fractal exponents [18]. Thereafter, the values of $\alpha_{\min }$ and $\alpha_{\max }$ are estimated to obtain the parameter $\Delta f(\alpha)$ as $\Delta f(\alpha)=f\left(\alpha_{\min }\right)-f\left(\alpha_{\max }\right) .|\Delta f(\alpha)|$ defines the undulation or instability of the system under study. The degree of undulation or instability is minimum for the smallest $\Delta f(\alpha)(\approx 0)$. 


\section{C. 'Stochastic' Multifractal Fields}

The seafloor bathymetry and the related backscatter images can be modeled taking into consideration a small number of (generally deterministic) or many (stochastic) degrees of freedom. In order to incorporate high degree of freedom and variability over a wide range of scales, stochastic approaches are preferred as they have infinite dimensional probability space. One way to characterize

the statistics of stochastic processes is to use its statistical moments. The statistical moments of a multifractal process is a power law that can be expressed as $\left\langle\phi_{\lambda}^{q}\right\rangle=\lambda^{K(q)}$, where $\phi_{\lambda}$ is the scale by scale conserved multifractal flux, $q$ is the order of the moment, $\lambda=L / l$ ( $L$ and $l$ represents largest and smallest scale in the data) is the scale ratio and $K(q)$ is a nonlinear convex function. $K(q)$ characterizes the scaling of the moments of the $\phi_{\lambda}$, hence it is called the 'moment scaling function'. With reference to the existence of stable attractive multifractal processes called 'universal multifractals' [19], $K(q)$ can be expressed as:

$$
K(q)=\frac{C_{1}}{\alpha-1}\left(q^{\alpha}-q\right)
$$

where $\alpha$ and $C_{1}$ are the basic parameters characterizing the scaling properties of the multifractal flux $\phi_{\lambda}$. The parameter $\alpha$ is the degree of multifractality and varies from 0 to 2, where $\alpha=0$ is the monofractal case and $\alpha=2$ is the log normal case. This parameter describes how rapidly the fractal dimensions of the sets at different thresholds vary as they leave the mean singularity. $C_{1}$ is the codimension parameter of the set. Low value of $C_{1}(\approx 0)$, implies that the field values are close to the mean. $C_{1}(>0)$ indicates that the region making the dominant contribution to the mean is a sparse fractal set such that the vast majority of the field doesn't contribute. The function $K(q)$ is related to the generalized dimension $D(q)$ (Section II B) as:

$$
D(q)=d-\frac{K(q)}{q-1}
$$

where $d$ is the dimension of the space (=2 here) [5].

The multiplicative process (the cascade) discussed in the preceding paragraph generates a scale by scale conserved multifractal flux $\phi_{\lambda}$ characterized by a moment scaling function $K(q)$. The spectrum of such a conserved flux has an exponent $\beta=1-K(2)<1$. In order to characterize the seafloor 
bathymetry and associated backscattering (having $\beta \approx 2$ ), Fractionally Integrated Flux (FIF) model [8] could be utilized. The FIF model of the multifractal flux provides the following statistics in relation to the intensity field $I_{\lambda}$ (bathymetry/backscatter) at scale ratio $\lambda$ as $I_{\lambda}=\phi_{\lambda} \lambda^{-H}$. Here the linear scaling $\lambda^{-H}$ corresponds to a fractional integral of order $H$. The parameter $H$ can be designated as a degree of smootheness where higher $H$ signifies smoother fields [20]. Characterization of seafloor topography using FIF model is difficult to distinguish as it involves a convolution due to the exponent $H$. Therefore, resorting to the use of 'trace moments' (that directly characterizes the conserved multifractal flux $\phi_{\lambda}$ ), is necessary so that the differentiation is possible. The 'trace moment' algorithm accessible on the website: http://www.physics.mcgill.ca/ eliasl/ has been used for this purpose.

The first step to obtain $\phi_{\lambda}$ from the intensity field involves removal of $\lambda^{-H}$ in the Eq. $I_{\lambda}=\phi_{\lambda} \lambda^{-H}$. This is equivalent to a filtering as in Fourier space with 'power law', which is a scale invariant smoothing. On elimination of $\lambda^{-H}$, only the underlying conserved multifractal flux $\phi_{\lambda}$ is retained. The next step is to examine the scaling of the statistical moments of $\phi_{\lambda}$. To this end, we normalize $\phi_{\lambda}$ so that the ensemble average of all the samples is $\left\langle\phi_{\lambda}\right\rangle=1$. Thereafter, spatial averaging is carried out over the sets (or squares) of size $l=L / \lambda$, the $q^{\text {th }}$ power is computed and the average over all data available is determined. It gives the moments of the normalized multifractal flux for a given value of $q$. This procedure is performed for different values of $q$ and $K(q)$ is evaluated from the logarithmic slopes (Fig.3). The multifractality of the intensity field can be validated with nonlinear $K(q)$. From the values of $K(q)$ the parameters $C_{1}$ and $\alpha$ are estimated as $C_{1}=K^{\prime}(1)$ and $\alpha=K^{\prime \prime}(1) / K^{\prime}(1)$ [21]. The values of $\alpha$ and $C_{1}$ combined with $\beta$ is used to estimate values of $H$ by the relation $\beta=1+2 H-K(2)$.

\section{STUDY RESULTS AND DISCUSSION}

\section{A. 'Strange' Attractors Based Technique}

As mentioned earlier, the degree of multifractality can be easily related to the width of the generalized dimension $\Delta D(q)$ and the $f(\alpha)$ spectrum. The computed $\Delta D(q)$ values get successively reduced from maximum to minimum, in the case of the backscatter blocks: Q19, J19, S23, F07, N25 and F20, associated with low, high, very low, moderate, nil, and very high seepages respectively [Fig. 4(a)]. Such reductions in $\Delta D(q)$ values indicate decrease in the degree of multifractality. Generally, low $\Delta D(q)$ values of bathymetry data blocks indicate comparatively reduced multifractality than the corresponding backscatter block [Fig. 4(b)]. However, gradual reduction of $\Delta D(q)$ values among the bathymetry data 
blocks show successive reduction in the degree of multifractality or monofractality (particularly in Q19 and S23 blocks) as $D(q)$ vs. $q$ curves are unvarying. Interestingly, the overall observation of the $D(q)$ vs. $q$ plots of backscatter as well as bathymetry data blocks imply similar construal for $f(\alpha)$ spectrum. The Q19 and S23 bathymetry blocks show single data point in the $f(\alpha)$ spectrum i.e., monofractality [Fig. 4(b)].

It is further observed that the shape parameters estimated from the $f(\alpha)$ spectrum also provide information about the multifractality [18]. The $W$ values of the four backscatter blocks, Q19, S23, J19 and F07, show gradual decrease in the degree of multifractality (heterogeneity) in a decreasing order. Though, Q19 and S23 blocks are located at a relatively shallower depth $(\approx 180 \mathrm{~m})$, they have low to very low backscatter strength indicating dominant multifractality (heterogeneity) [Fig. 5(a)]. Intriguingly, bathymetry blocks with negligible $W$ values display relatively reduced degree of multifractality as compared to the corresponding backscatter blocks. This may be due to the presence of shell materials along with coarse sediments and the changes in the seafloor roughness at the textural level caused by bottom currents (Fig. 1) [9], [11]. Such changes at the textural level can only be notably detected in the backscatter data as compared to the bathymetry data.

The estimated $B$ values using backscatter image blocks reveal that all the blocks are positive or right skewed except S23. Among them, the blocks J19 and F20, located away from fault regime (Fig. 1), possess higher $B$ values indicating dominance of higher fractal exponents [Fig. 5(a)]. Whereas S23 block possess a negative value of $B$ showing left skewed spectrum i.e., the dominance of lower fractal exponents. The estimated $B$ parameters of all the bathymetry blocks are significantly low and negative (left skewed), indicating that the distributions are dominated with lower fractal exponent.

The positive values of the parameter $\Delta f(\alpha)$, are seen successively diminishing in the case of backscatter image blocks J19, F07, F20, Q19, N25 and S23, suggesting reduction in the undulations or instability at the textural level [Fig. 5(a)]. However in the case of bathymetry image blocks, low negative values of $\Delta f(\alpha)$ are observed in all the six blocks. The variability of the three parameters $[W, B$, and $\Delta f(\alpha)]$ of the backscatter blocks is more conspicuous than their corresponding parameters of the bathymetry blocks, indicating dominant fine scale undulations in the backscatter as compared to the depth data. The scatter plots [Fig. 5(b)] of the three parameters affirm the location wise study results using the 'strange' attractor technique. 


\section{B. 'Stochastic' Multifractal Field based Technique}

In our analyses, the universal form (determined from Eq.3 based on the estimated $\alpha$ and $C_{1}$ ) fits the empirical $K(q)$ (determined from the logarithmic slopes of trace moments) quite well.

A 'multifractal phase transition' [21] is observable in the plot (Fig. 3) of empirical and theoretical $K(q)$ curves, indicating that the measured moments will only have the theoretical $K(q)$ for $q$ below a critical moment $q_{c}$. Beyond $q_{c}$ there is a multifractal phase transition where $K(q)$ becomes asymptotically linear for $q \geq q_{c}>1.5$ (a sample size-dependent effect corresponding to the domination of the statistics by the largest flux values present). Indeed, for $q<1.5$, the deviations from the universal form are negligible.

The $\alpha$ values of the bathymetry and backscatter blocks show identical trend $(\approx 2)$ expressing similar degree of multifractality excluding Q19 and S23 of the bathymetry blocks [Fig. 5(c)]. The low $\alpha$ values of the two blocks are well corroborated with the results of the other formalism signifying monofractality. The $C_{1}$ values of the backscatter and bathymetry blocks are found to be varying between 0.058 and 0.091 and between 0.038 and 0.690 respectively. Excluding the S23 bathymetry block $\left(C_{1}=\right.$ 0.690), the lower values of $C_{1}$ attributed to the remaining blocks, indicate that the field values are close to the mean values. Fluctuations in higher values of $H$ between 0.636 and 0.706 are observed in the backscatter image blocks, except in the N25 block (0.412) having no evidence of seepages. Whereas, lower $H$ values $(0.255-0.480)$ are observed in the bathymetry as compared to the backscatter blocks at the same location. The estimated $H$ value of N25 bathymetry block is the lowest.

Cluster analyses output [Fig. 5(d)] of $H$ vs. $\alpha$ reveals a uniform $\alpha$ in the backscatter and bathymetry blocks except in the Q19 bathymetry block. However, the $H$ value of the bathymetry block S23, does not subsist owing to the isotropic field condition as mentioned in p. 549 of [8]. Remarkably the clustering tendency around the high $\alpha(\approx 2)$ and low $C_{1}$ of the bathymetry and backscatter data, show extremely close relationship among, the six backscatter and four bathymetry blocks [Fig. 5(d)]. However both Q19 and S23 bathymetry blocks possess comparable $\alpha$ values and relatively higher $C_{1}$ values. The $C_{1}(0.690)$ value of the S23 bathymetry block espouse the setting wherein the depths at specific locations (i.e., center of the pockmark) are higher as compared to the rest of the locations within the block. In this study, the 'stochastic' multifractal based technique show no significant variation in $\alpha$ and $C_{1}$ of the backscatter field data, except parameter $H$. 


\section{CONCLUSIONS}

Two multifractal formalisms were applied to the backscatter and similar located bathymetry blocks to characterize the pockmark seepage associated seafloor (WCMI). The first formalism employs data analyses based on the 'strange' attractor technique, and the other approach is designed as 'stochastic' multifractal field. The outcome of the application of the 'strange' attractor technique, $\Delta D(q)$ and the $f(\alpha)$ spectrum related shape parameters $[W, B$ and $\Delta f(\alpha)]$ reveal multifractal character of the six backscatter blocks. The variability of the estimated shape parameters is more apparent in the backscatter as compared to the corresponding bathymetry blocks. This can be related to the interpretation of $\Delta D(q)$, and $f(\alpha)$ parameters of the ECG signal of a healthy and a diseased heart [22]. The above referred multifractality aspects support the fact that greater the data heterogeneity, higher would be the system stability. Characteristically, higher stability can be realized when $|\Delta f(\alpha)|$ is low. Appropriately Q19 and S23 backscatter blocks reveal low $|\Delta f(\alpha)|$ values. The stability attributed to the blocks could be ascribed to their location in shallow depth $(\approx 180 \mathrm{~m})$ as compared to the rest of the blocks. Moreover, the above blocks possess coarse seafloor sediments having dominant shell materials, and are influenced by the monsoonal bottom currents, resulting in greater heterogeneity [9].

The three computed parameters $\left(\alpha, C_{1}\right.$, and $\left.H\right)$ from bathymetry and backscatter blocks using 'stochastic' multifractal formalism show almost similar degree of multifractality with the exception of Q19 and S23 bathymetry blocks. The low values of the two blocks are in sound corroboration as they show monofractality. Generally the level of the $C_{1}$ values of backscatter data blocks are found to be higher (0.058-0.091) than the bathymetry $(0.038-0.690)$. In the case of backscatter image blocks, higher $H$ values (0.636-0.706) are observed except in $\mathrm{N} 25$ block (0.412) having no seepages. On the other hand, lower $H$ values $(0.255-0.480)$ are observed in the bathymetry blocks in relation to the backscatter. The advantage of the 'stochastic' multifractal technique is that it provides three distinct parameters, with which it is easier to comprehend the multifractality aspects of the seafloor than the 'strange' attractor based $f(\alpha)$ spectrum related shape parameters. Cheng and Agterberg [23] had made assessment between the interrelationships of the two methods (akin to the ones we have used here) and had suggested the aptness of the multifractal spectrum $f(\alpha)$ over the codimension function $C_{1}$. The two multifractal techniques utilized in our work is a first-time attempt to analyze the high resolution MBES backscatter and bathymetry data. The present investigation employing both the methods ascertain an important finding, however further interest is required to expound the techniques. 


\section{ACKNOWLEDGEMENTS}

The authors are thankful to the Director, NIO, for permitting the publication of this work. Financial support from NIOT (Chennai) and MoES (New Delhi) is duly acknowledged. KH expresses his thanks to CSIR for the grant of fellowship. The authors express their gratitude to the editors and the two reviewers of IEEE GRSL for their guidance to improve the quality of this manuscript. This is NIO contribution XXXX.

\section{REFERENCES}

[1] B. B. Mandelbrot, 'Multifractal measures, especially for the geophysicist,' PAGEOPH, vol. 131, no. 1-2, pp. 5-42, Jan-Feb.1989.

[2] H. G. E. Hentschel and I. Procaccia, 'The infinite number of generalized dimensions of fractals and strange attractors,' Physica D: Nonlinear Phenomena, vol. 8, no. 3, pp. 435-444, Sep. 1983.

[3] S. Lovejoy, D. Schertzer, V. Allaire, T. Bourgeois, S. King, J. Pinel, and J. Stolle, 'Atmospheric complexity or scale by scale simplicity?,' Geophys. Res. Lett., vol. 36, no. 6, pp. 1-6, Jan. 2009.

[4] P. C. Ivanov, L. A. Nunes Amaral, A. L. Goldberger, S. Havlin, M. G. Rosenblum, Z. R. Struzik, and H. E. Stanley, 'Multifractality in human heartbeat dynamics,' Nature, vol. 399, no. 6735, pp. 461-465, Jun. 1999.

[5] L. Seuront, Fractals and Multifractals in Ecology and Aquatic Science. New York: CRCPress, 2010.

[6] T.C. Halsey, M.H. Jensen, L.P. Kadanoff, I. Procaccia, and B.I. Shraiman, 'Fractal measures and their singularities: The characterization of strange sets,' Phys. Rev. A., vol. 33, no. 2, pp. 11411151, Feb. 1986.

[7] D. Schertzer and S. Lovejoy, 'Physical modeling and analysis of rain and clouds by anisotropic scaling of multiplicative processes,' J. Geophys. Res., vol. 92, no. D8, pp. 9693-9714, Aug. 1987.

[8] J.-S. Gagnon, S. Lovejoy, and D. Schertzer, 'Multifractal earth topography,' Nonlin. Processes Geophys., vol. 13, no. 5, pp. 541-570, Oct. 2006.

[9] S. Dandapath, B. Chakraborty, S.M. Karisiddaiah, A. Menezes, G. Ranade, W. Fernandes, D. K. Naik, and K.N. Prudhvi Raju, 'Morphology of pockmarks along the western continental margin of India: employing multibeam bathymetry and backscatter data,' Mar. Petrol. Geol., vol. 27, no. 10, pp. 2107-2117, Dec. 2010.

[10] L. Seuront and N. Spilmont, 'Self-organized criticality in intertidal microphytobenthos patch patterns,' Physica A, vol. 313, no. 3-4, pp. 513-539, Apr. 2002. 
[11] S. Dandapath, B. Chakraborty, N. Maslov, S.M. Karisiddaiah, D. Ghosh, W. Fernandes, and A. Menezes, 'Characterization of seafloor pockmark seepage of hydrocarbons employing fractal: A case study from the western continental margin of India,' Mar. Petrol. Geol.,vol. 29, no. 1, pp. 115-128, Jan. 2012.

[12] C. De and B. Chakraborty, 'Model-based acoustic remote sensing of seafloor characteristics,' IEEE Trans. Geosci. Remote Sens., vol. 49, no. 10, pp. 3868-3877, Oct. 2011.

[13] K. Haris, B. Chakraborty, C. De, R. G. P. Desai, and W. A. Fernandes, 'Model-based seafloor characterization employing multi-beam angular backscatter data - A comparative study with dual-frequency single beam,' J. Acoust. Soc. Amer., vol. 130, no. 6, pp. 3623-3632, Dec. 2011.

[14] B. Chakraborty, V. Mahale, C. de Sousa, and P. Das, 'Seafloor classification using echowaveforms: a method employing hybrid neural network architecture,' IEEE Geosci. Remote Sens. Lett., vol. 1, no. 3, pp. 196-200, Jul. 2004.

[15] C. De and B. Chakraborty, 'Acoustic characterization of seafloor sediment employing a hybrid method of neural network architecture and fuzzy algorithm,' IEEE Geosci. Remote Sens. Lett., vol. 6, no. 4, pp. 743-747, Oct. 2009.

[16] A. B. Chhabra and R. V. Jensen, 'Direct determination of the $f(\alpha)$ singularity spectrum,' Phys. Rev. Lett., vol. 62, no. 12, pp. 1327-1330. Mar. 1989.

[17] A. Szczepaniak, and W. M. Macek, 'Asymmetric multifractal model for solar wind intermittent turbulence,' Nonlin. Processes Geophys., vol 15, no. 4, pp. 615-620, Jul. 2008.

[18] L. Telesca, G. Colangelo, V. Lapenn, and M. Macchiato, 'Monofractal and multifractal characterization of geoelectrical signals measured in southern Italy,' Chaos. Solition Fract., vol. 18 , no. 2, pp. 385-399, Oct. 2003.

[19] L. Seuront, F. Schmitt, Y. Lagadeuc, D. Schertzer, and S. Lovejoy, 'Universal multifractal analysis as a tool to characterize multiscale intermittent patterns: example of phytoplankton distribution in turbulent coastal waters,' J. Plankton Res., vol. 21, no.5, pp. 877-922, May. 1999.

[20] S. Lovejoy and D. Schertzer, 'Scaling and multifractal fields in the solid earth and topography,' Nonlin. Processes Geophys., vol. 14 , no. 4, pp. 465-502, Aug. 2007.

[21] J. Stolle, S. Lovejoy and D. Schertzer, 'The stochastic multiplicative cascade structure of deterministic numerical models of the atmosphere,' Nonlin. Processes Geophys., vol. 16, no. 5, pp. 607-621, Oct. 2009.

[22] H. E. Stanley, L. A. N. Amral, A. L. Goldberger, S. Havlin, P. Ch. Ivanov, and C-K. peng, 'statistical physics and physiology: monofractal and multifractal approaches,' Physica A., vol. 270, pp. 309-324, 1999.

[23] Q. Cheng and F. P. Agterberg, 'Comparison between two types of multifractal modeling,' Math. Geol., vol. 28 no. 8, pp.1001-1015, Aug. 1996. 


\section{Figure captions:}

Fig. 1. (a) Study blocks including some of the main structural features of the region of the western continental margin of India (WCMI). (b) Backscatter map of the study area showing 160 to $320 \mathrm{~m}$ isobaths with $20 \mathrm{~m}$ interval. Pockmarks are indicated by crossed circles. Black, blue and red color mark represents circular, elliptical and elongated pockmarks respectively. The dashed lines indicate location of the identified faults. The black arrows show bottom current directions. Solid colored squares represent the sediment types.

Fig. 2. Schematic representation of (a) generalized correlation dimension function $D(q)$ for estimation of the parameter $\Delta D(q)$ and (b) multifractal spectrum $f(\alpha)$ for assessment of the three multifractal parameters i.e., width of the spectrum $W$, degree of asymmetry $B$ and stability of the spectrum $\Delta f(\alpha)$ based on 'strange' attractor formalism.

Fig. 3. The scaling behavior of the statistical moments of the two representative backscatter and bathymetry image data is illustrated here by the straightness of the Log/log curves of the normalized 'trace moment' $(M)$ as functions of the scale ratio $\lambda=L / l$. The values of the exponent $q$ of each trace moments are varied between 0 -2. The linear deviation of dashed curves for $q \geq q_{c}>1.5$ is indicative of a multifractal phase transition.

Fig. 4. Block wise generalized correlation dimension $D(q)$ and multifractal spectrum $f(\alpha)$ plots for (a) backscatter strength, and (b) bathymetry data of the study blocks.

Fig. 5. Results obtained using the two multifractal formalisms. (a) Block wise multifractal spectrum $f(\alpha)$ related shape parameters estimated using 'strange' attractor formalism. (b) Scatter plots of the three multifractal parameters $[W, B$, and $\Delta f(\alpha)]$. The bathymetry blocks are encircled. (c) Block wise multifractal parameters estimated using 'stochastic' multifractal formalism. (d) Scatter plots of the three multifractal parameters $\left(\alpha, C_{1}\right.$ and $\left.H\right)$. The solid and hollow shapes represent different study blocks as shown in the legend. 


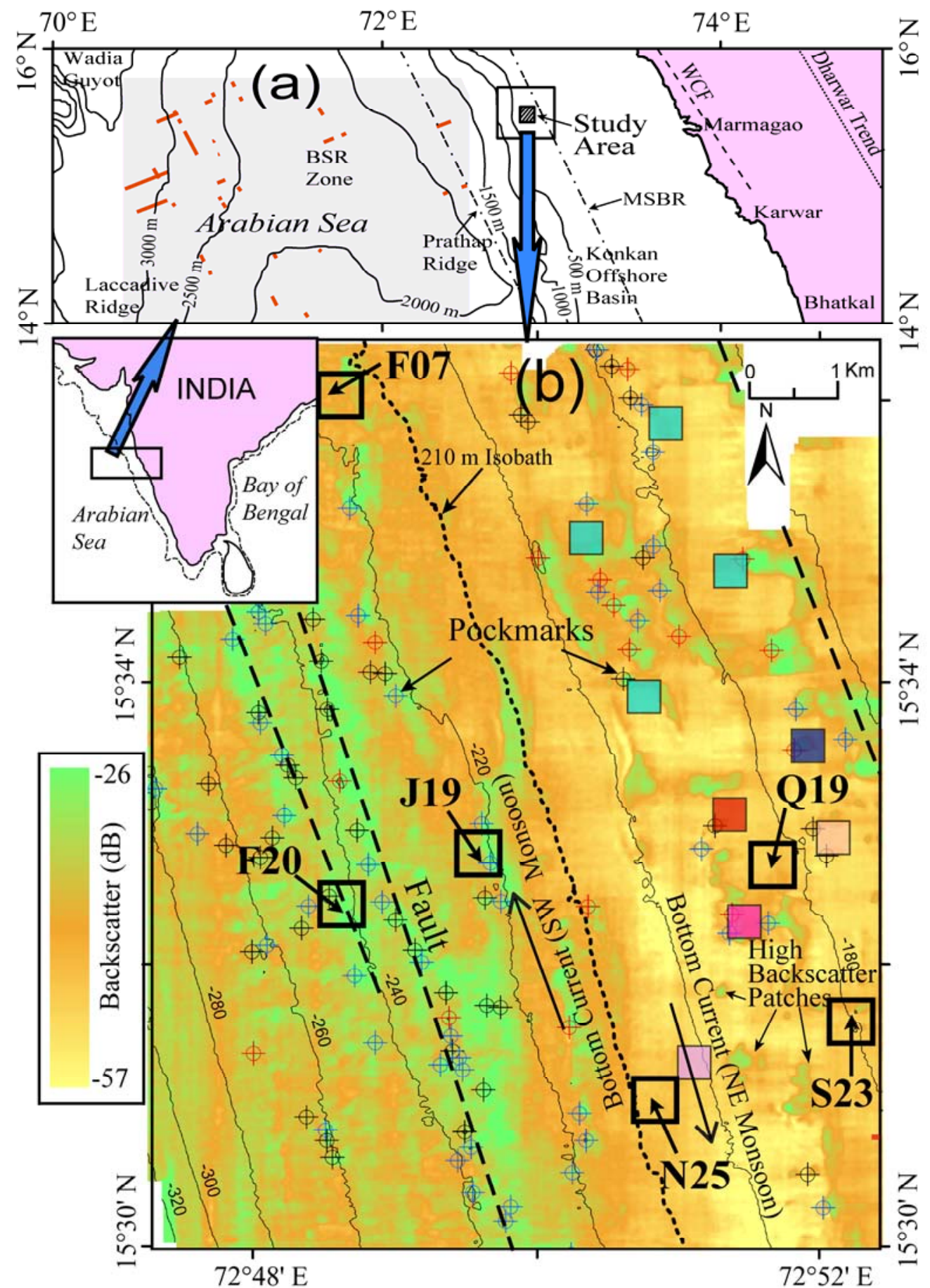

$\square$ Clayey sand and less shell material Clayey sand and abundant shell material Sandy material $\square$ Study blocks Sandy material with less shells Sandy material with abundant shells Abundant shells with sandy material

Figure 1 

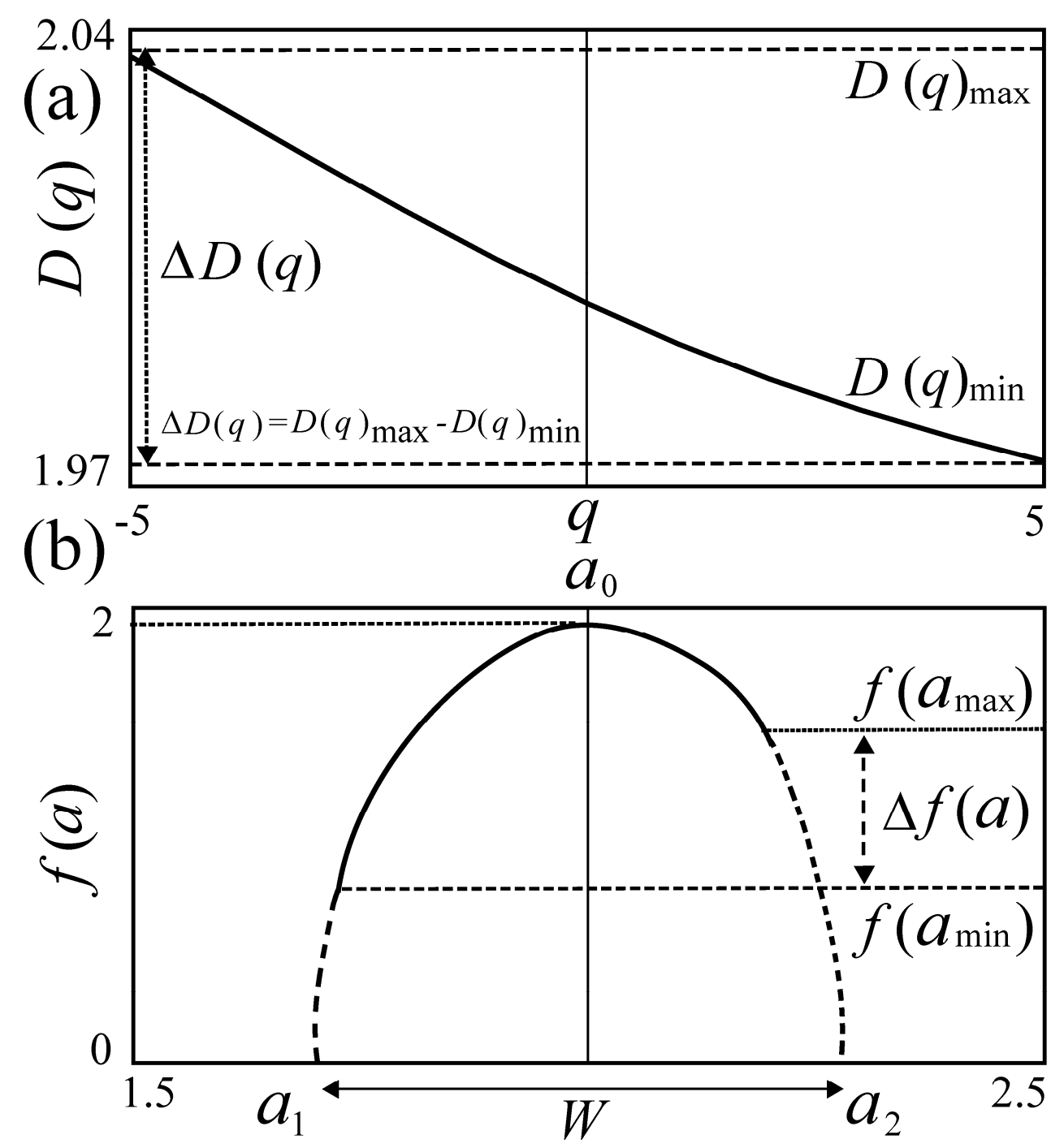

Width of the spectrum ' $W$ ' $=a_{2}-a_{1}$

$$
\text { where, } f\left(a_{1}\right)=f\left(a_{2}\right)=0
$$

Stability of the spectrum ' $f(a)^{\prime}=f\left(a_{\min }\right)-f\left(a_{\max }\right)$

Degree of asymmetry ' $B^{\prime}=\frac{\left(a_{2}-a_{0}\right)-\left(a_{0}-a_{1}\right)}{\left(a_{2}-a_{1}\right)}$

Figure 2 

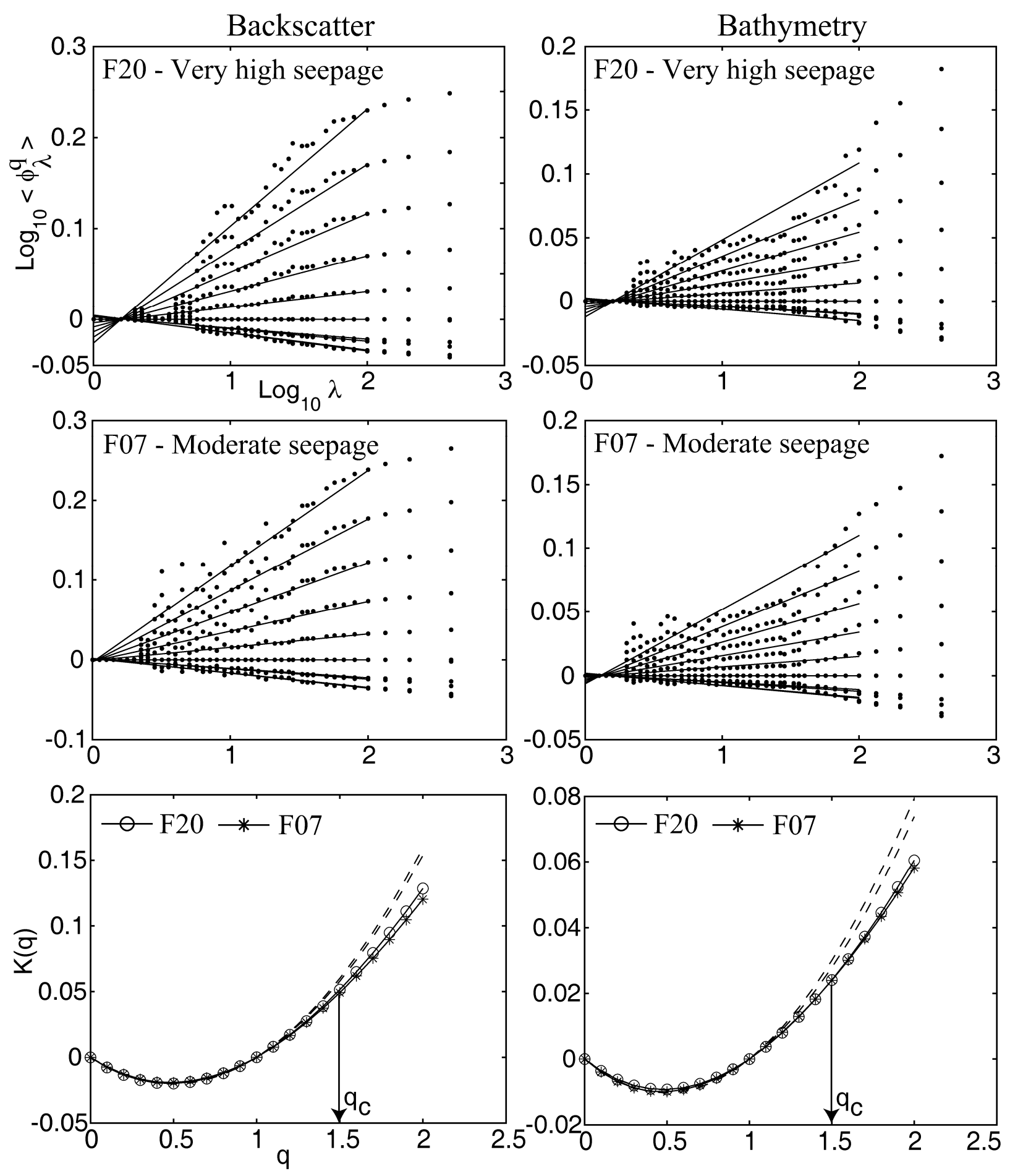

Figure 3 
(a)

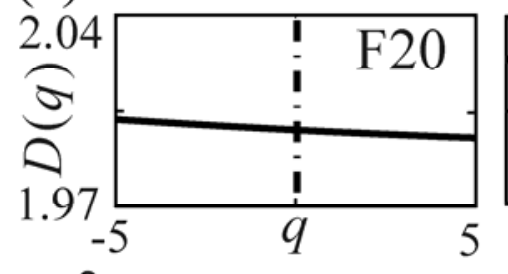

Backscatter blocks
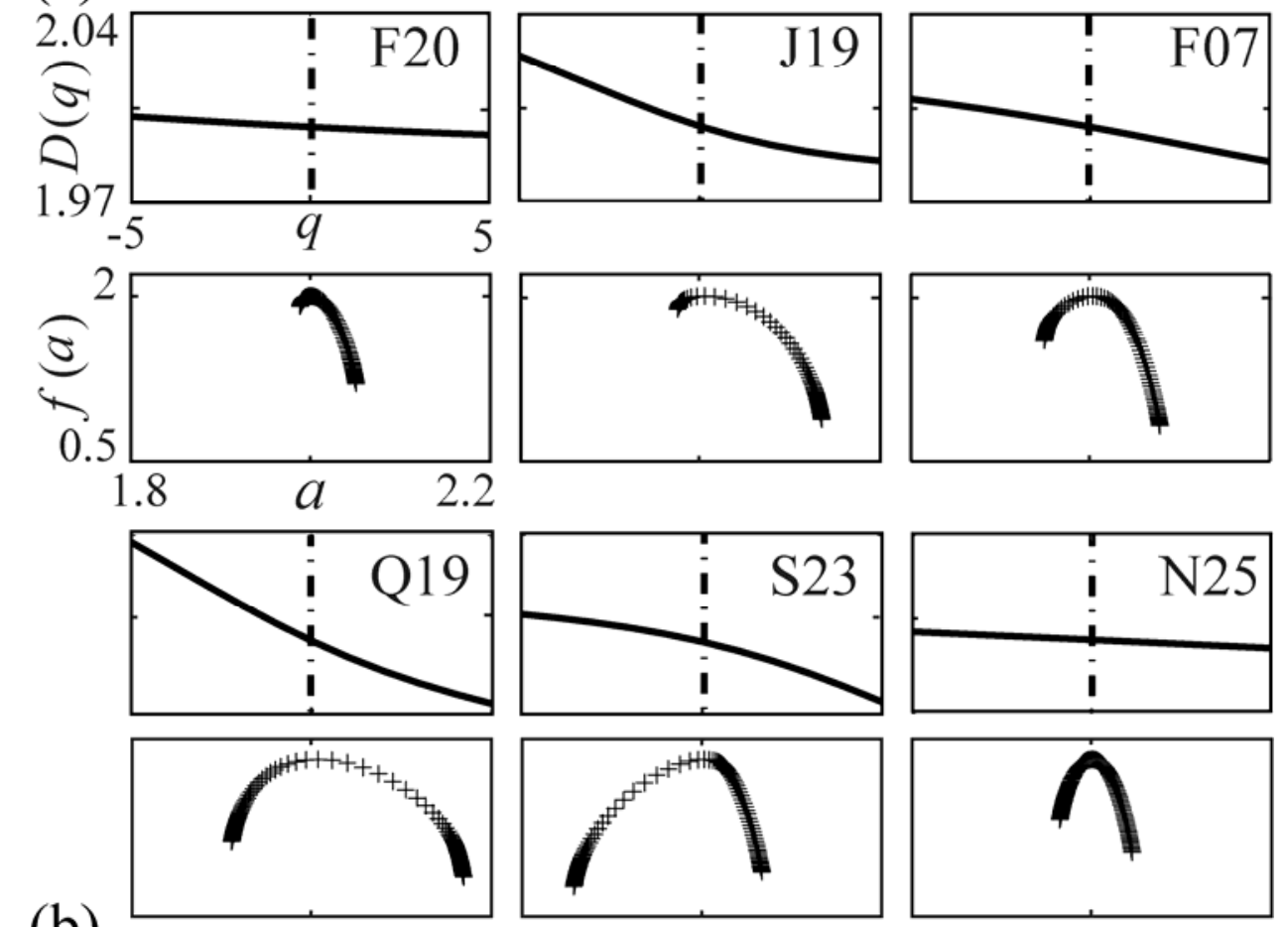

(b)
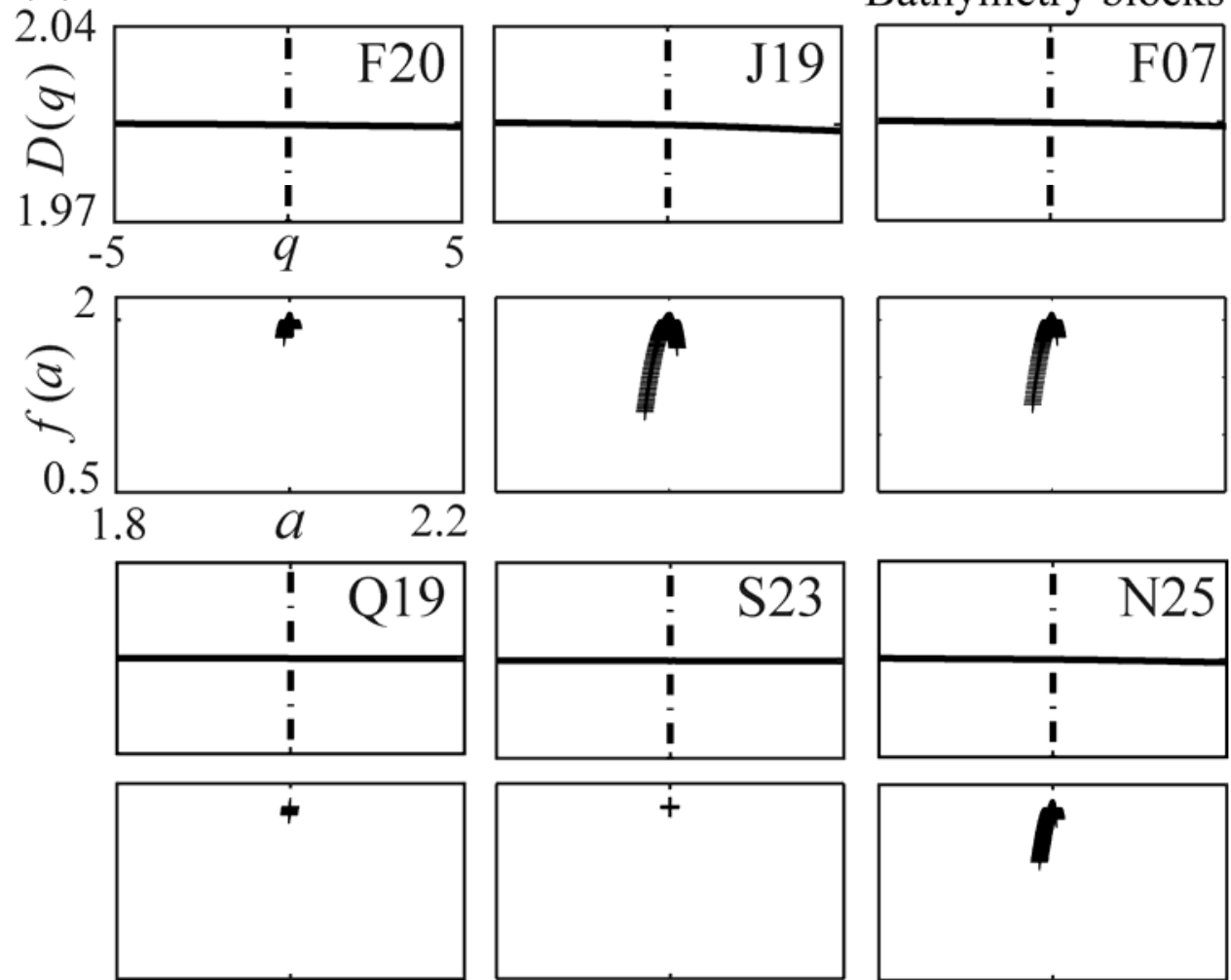
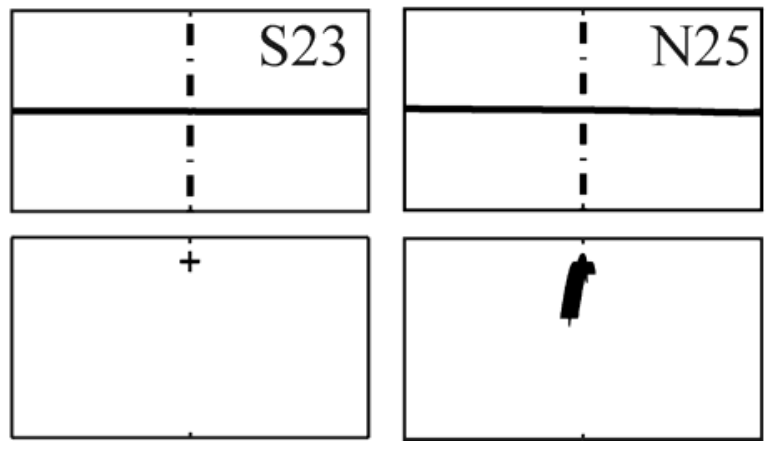

Figure 4 


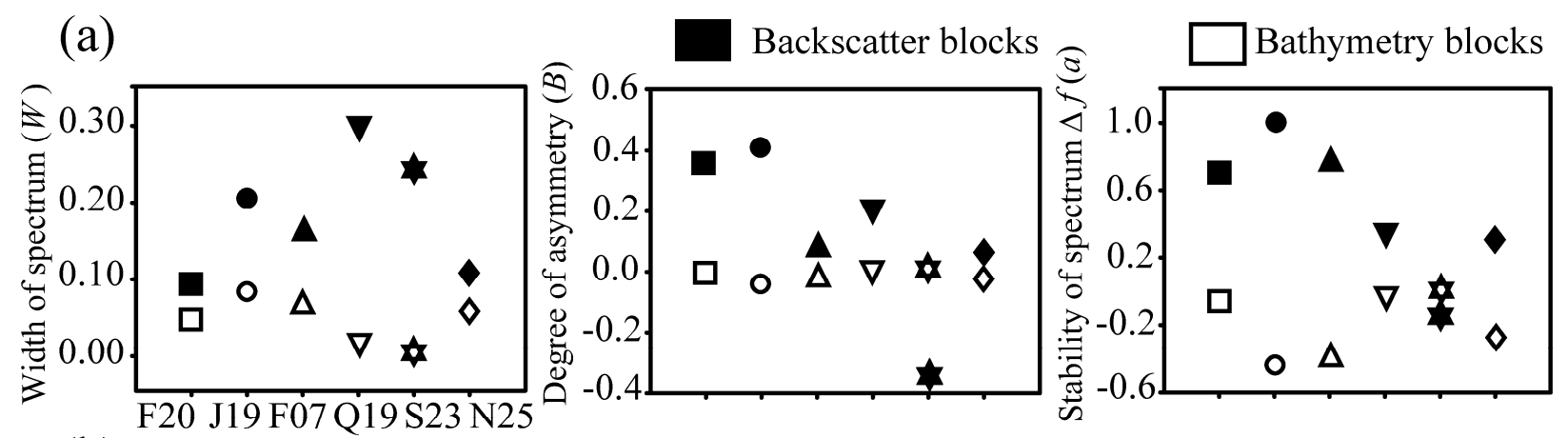

(b) Block
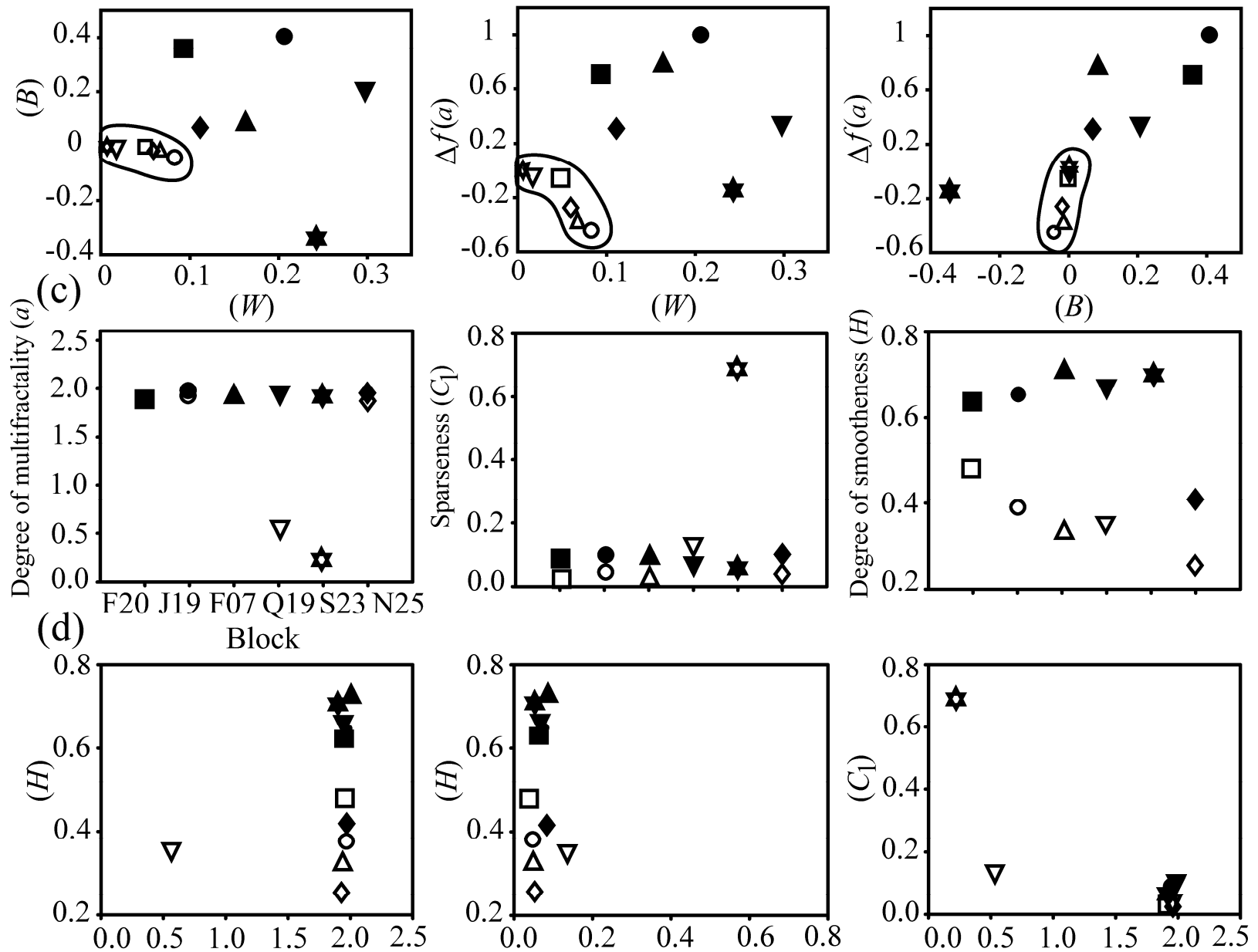

(a)

$\left(C_{1}\right)$

(a)

\begin{tabular}{llllllllllll}
\hline & F20 & $\circ$ & J19 & $\Delta$ & F07 & $\nabla$ & Q19 & $\star$ & S23 & $\diamond$ & N25
\end{tabular}

Figure 5 


\begin{tabular}{|c|c|c|c|c|c|c|}
\hline $\begin{array}{l}\text { Sample } \\
\text { blocks }\end{array}$ & F20 & J19 & F07 & Q19 & S23 & N25 \\
\hline Seepage type & Very high & High & Moderate & Low & Very low & No evidence \\
\hline $\begin{array}{c}\text { Mean } \\
\text { backscatter } \\
\text { strength }(\mathrm{dB}) \\
\text { (max to } \min ) \\
\end{array}$ & $\begin{array}{c}-31.58 \\
(-27.46 \text { to }- \\
35.71)\end{array}$ & $\begin{array}{c}-34.40 \\
(-27.50 \text { to }- \\
42.62)\end{array}$ & $\begin{array}{c}-38.99 \\
(-33.84 \text { to }- \\
42.84)\end{array}$ & $\begin{array}{c}-38.96 \\
(-36.18 \text { to }- \\
46.78)\end{array}$ & $\begin{array}{c}-43.28 \\
(-37.24 \text { to }- \\
45.11)\end{array}$ & $\begin{array}{c}-42.62 \\
(-41.36 \text { to }- \\
44.19)\end{array}$ \\
\hline $\begin{array}{l}\text { Backscatter } \\
\text { image of } \\
\text { sample } \\
\text { blocks }\end{array}$ & & & & & 8 & \\
\hline $\begin{array}{c}\text { Bathymetry } \\
\text { of the sample } \\
\text { blocks } \\
\text { (contour } \\
\text { interval } \\
0.5 \mathrm{~m} \text { ) }\end{array}$ & 㢳 & & a & & & \\
\hline
\end{tabular}

Table 1: Backscatter (in digital number: 0-255) and bathymetry seafloor seepage blocks selected from the Fig.1 for the present investigation. 